\title{
Microstrip band-stop filter based on double negative metamaterial
}

\author{
Badr Nasiri' ${ }^{1}$, Ahmed Errkik ${ }^{1}$, Jamal Zbitou ${ }^{2}$ \\ ${ }^{1}$ LMIET, FST of Settat, Hassan First University, Settat, Morocco \\ ${ }^{2}$ LABTIC, ENSA of Tangier, Abdelmalek Essaadi University, Tangier, Morocco
}

\begin{tabular}{l} 
Article Info \\
\hline Article history: \\
Received Jun 9, \\
Revised Sep 21, \\
Accepted Oct 14, \\
\hline Keywords: \\
Band-stop \\
Double negative \\
Filter \\
Metamaterial \\
Microstrip
\end{tabular}

\section{Corresponding Author:}

Badr Nasiri

LMIET, FST of Settat, Hassan First University

FST de Settat, Km 3, B.P: 577 Route de Casablanca, Settat, Morocco

Email: b.nasiri@uhp.ac.ma

\begin{abstract}
In this work, we present a novel miniature band stop filter based on double negative metamaterial, this circuit is designed on a low-cost substrate FR-4 of relative permittivity 4.4 and low tangential losses 0.002 . The proposed filter has a compact and miniature size of $15 \mathrm{~mm}$ in length and $12 \mathrm{~mm}$ in width without the $50 \Omega$ feed lines. The resonator was studied and analyzed with a view to achieving a band-stop behavior around its resonant frequency. The band-stop characteristics are obtained by implementing the metamaterial resonator on the final structure. The obtained results show that this microstrip filter achieves fractional bandwidth of $40 \%$ at $2 \mathrm{GHz}$. Furthermore, excellent transmission quality and good attenuation are achieved. This filter is an adequate solution for global system for mobile communications (GSM).
\end{abstract}

This is an open access article under the CC BY-SA license.

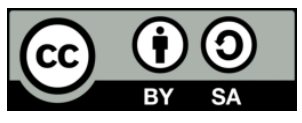

\section{INTRODUCTION}

The microstrip circuits have very important applications in wireless and radio frequency (RF) systems because of the outstanding virtues of simple integration with the microwave components and other lumped elements [1]-[3]. More often than not, the planar filter kind is extensively used in modern wireless systems because of their significant role by suppressing harmonics and spurious signals [4]-[7]. The spreading of this type of circuits in these systems gives great attention to the design of a miniature circuit filter has excellent performances [8]-[11]. However, achieving these goals simultaneously is not available by using conventional methods. So, the advanced technical methods which are used to obtain size reduction and electrical performances improvements such as microstrip line with etched spiral resonators, metamaterial and defected ground structure have been studied in [12]-[16].

Recently, subwavelength resonators are extensively implemented in microwave circuits in order to design a miniature filter, antenna, and coupler [17]-[20]. These engineered materials are characterized by unusual electromagnetic proprieties, their effective constitutive parameters have a negative value that is not available in ordinary materials [21], [22]. these peculiarities allow us to get resonator structures are so smaller than the wavelength of the interacting signals which have many useful advantages of radiofrequency devices in term of circuit size and electrical performances [23]-[25].

This paper describes a new miniature band-stop filter based on metamaterial resonator unit cell. This proposed back surface field (BSF) has a good transmission quality in the first and second pass-band and an excellent rejection level in the stop-band. Moreover, its circuit is characterized by the small size, the easy 
fabrication, and low cost. Furthermore, the proposed filter is a good solution for global system for mobile (GSM), long term evolution (LTE) and radio frequency identification (RFID) applications.

\section{DESIGN PROCEDURES}

With a view to achieve some objectives in terms of final circuit size and band stop filter electrical responses, a novel metamaterial unit cell has been studied analyzed and implemented. The concept of metamaterial was started by the physicist Victor Georgievich Veselago at the end of 1967 [23]. After 30 years, Pendry et al. [24], [25] are succeeded to propose a medium with negative permittivity and negative permeability. By using the split-ring resonators and metallic wires, David R. Smith could fabricate a medium media of metamaterial in 2001[26].

After the chosen of the flame resistant 4 substrate FR4, the unit cell parameters are chosen following numerous steps of optimization and analysis with a view to obtain and reach a desired resonant frequency which will be used as an operating frequency of the proposed circuit. The studied resonator is shown in Figure 1, and its simulated scattering parameters are displayed in Figure 2.

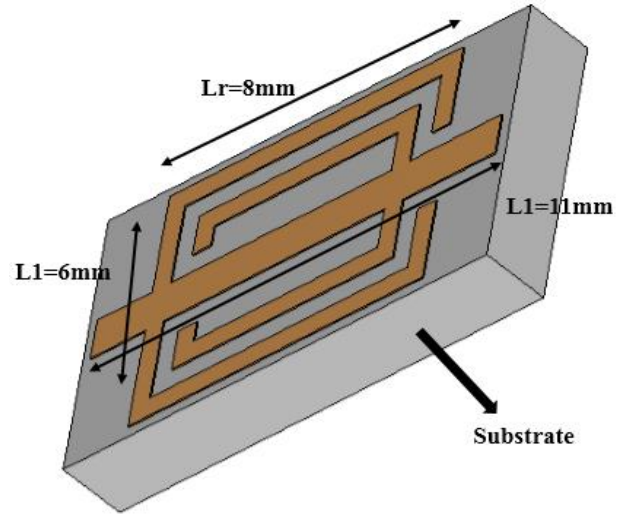

Figure 1. Metamaterial unit cell

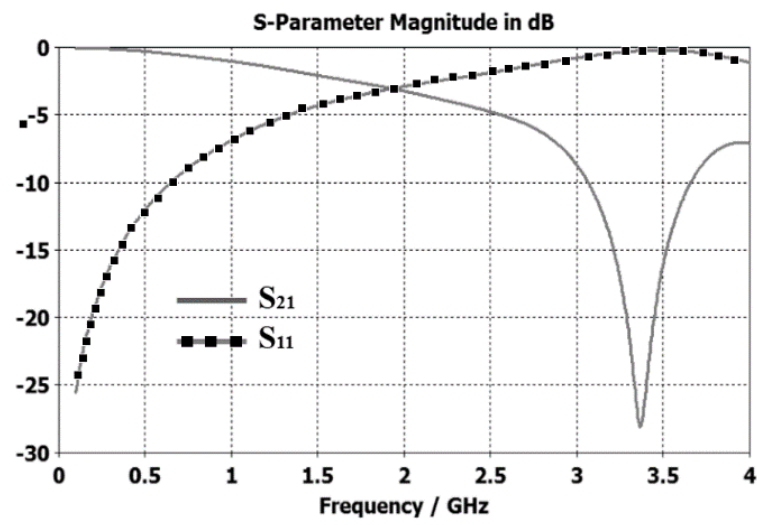

Figure 2. Metamaterial frequency responses

After the chosen of the optimized geometrical parameters of the metamaterial resonator and the obtainment of the desired range of frequencies. The investigation of unusual electromagnetic characteristics of this designed resonator was performed by using the s-parameter retrieval method. The effective permittivity, permeability, and refractive index are related to the S11 and S12 by the (1)-(4).

$$
\begin{aligned}
& n=\frac{1}{K_{0}} \cos ^{-1}\left[\frac{1}{2 S_{21}}\left(1-S_{11}^{2}+S_{21}^{2}\right)\right] \\
& Z= \pm \sqrt{\frac{\left(1+S_{11}\right)^{2}+S_{21}^{2}}{\left(1-S_{11}\right)^{2}+S_{21}^{2}}} \\
& \text { Eeff }=\frac{n}{Z} \\
& \mu e f f=Z n
\end{aligned}
$$

Figure 3 shows the effective medium parameters and refractive index of the proposed double negative metamaterial unit cell. As might be seen, this resonator provides a negative refractive index, negative real permeability, and negative real permittivity close to its resonant frequency. The design steps of the final circuit, as well as the geometrical parameters of the proposed band stop filter, are displayed in Figure 4. The structure is composed of two $50 \Omega$ microstrip lines connected with modified element microstrip line. Then, the proposed resonator is added and located in the center of the microstrip circuit. The optimized filter is very simple so that the manufacturing problems and complexity can be reduced. This filter has a miniature size of $12 \times 21 \mathrm{~mm}^{2}$ in comparison to its operating frequency.

The proposed structure without a metamaterial resonator is simulated with a view to understanding the influence of the chosen metamaterial unit cell. It is evident from Figure 5 that there is a normal and 
simple transmission and that no rejected band is remarked. To meet the requirement of the stop band characteristics of proposed circuit in terms of size and electrical performance, several simulations have been carried out, studied, and analyzed in order to choose the best one that meets the specifications of the desired band stop filter. The proposed BSF structure is presented in Figure 6.

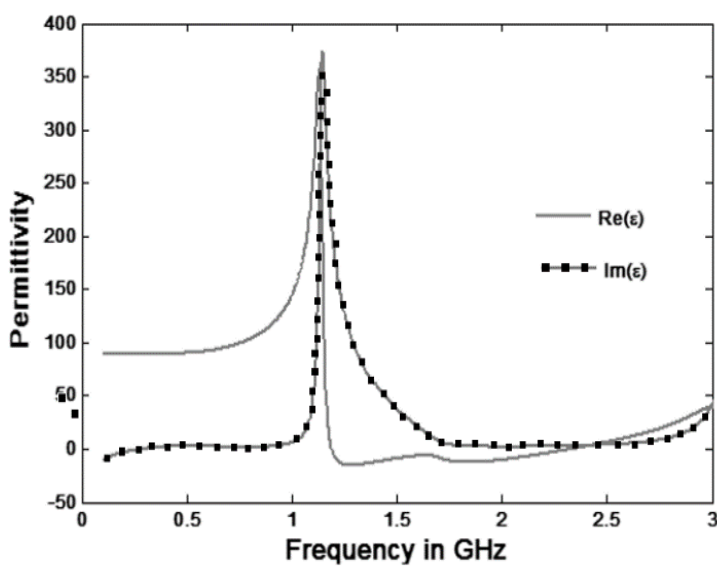

(a)

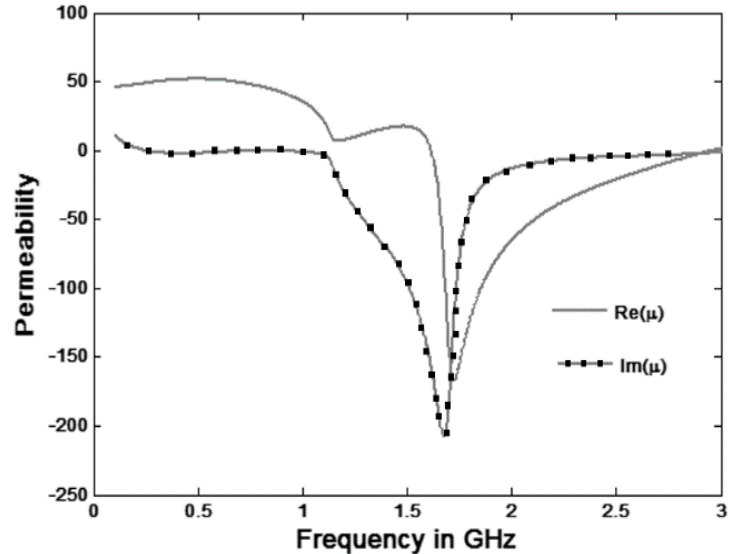

(b)

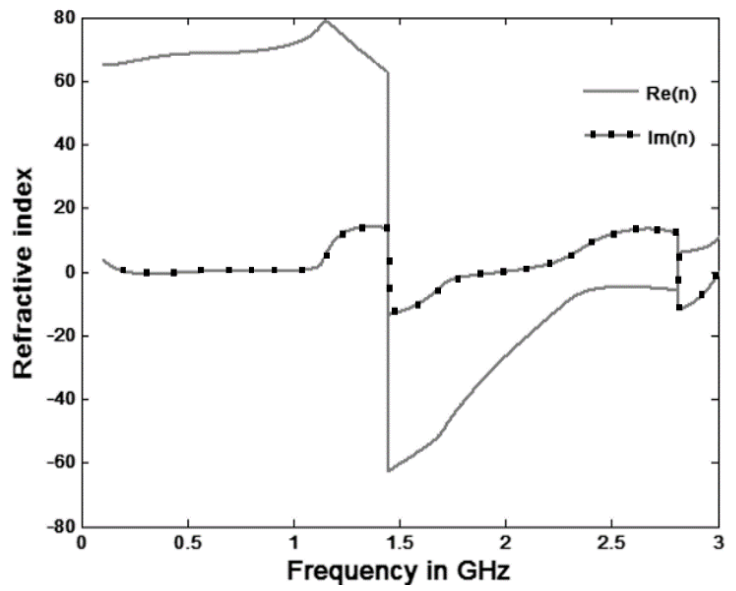

(c)

Figure 3. The effective medium parameters (a) permittivity, (b) permeability, and (c) refractive index

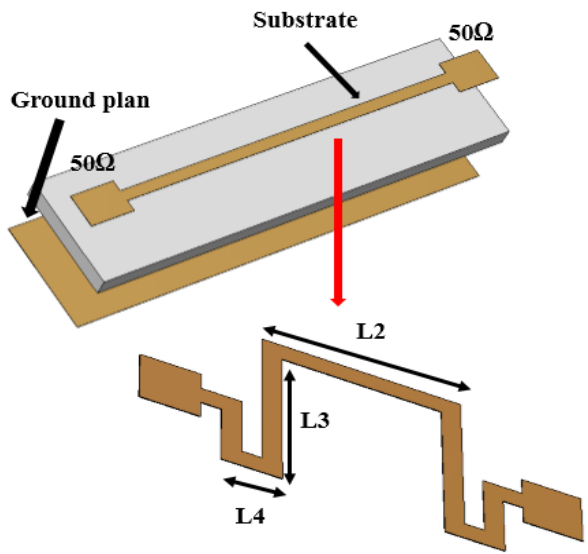

Figure 4. Design steps of BSF

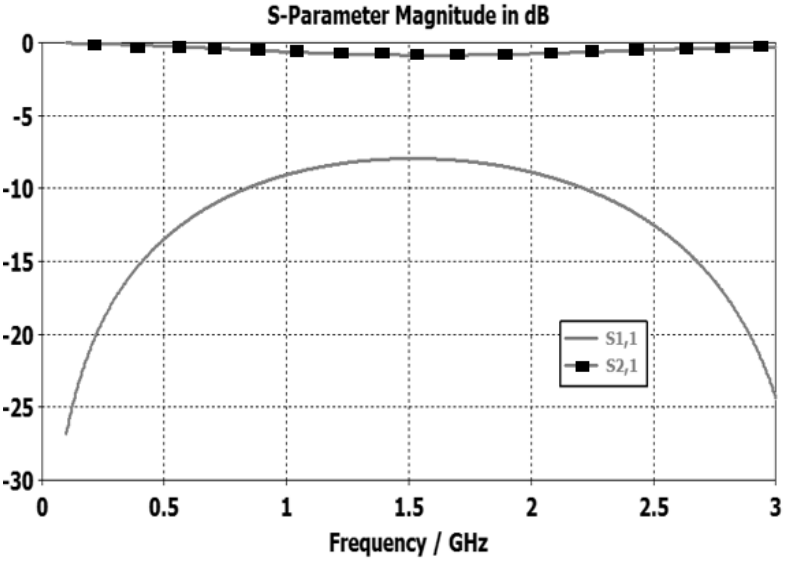

Figure 5. Simulated results without a metamaterial resonator 


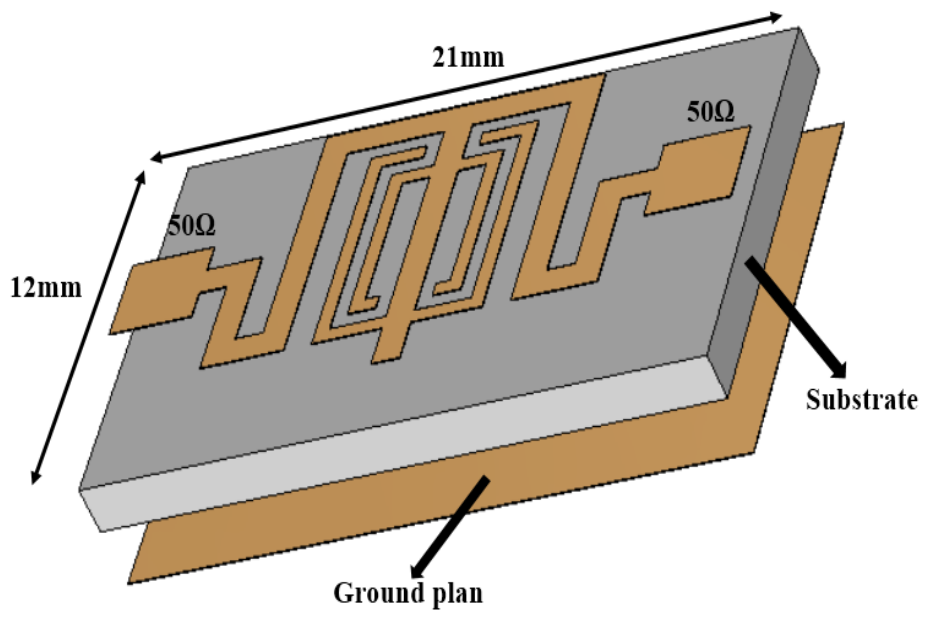

Figure 6. Proposed band stop filter

\section{RESULTS AND DISCUSSION}

Figure 7 illustrates the S-parameters results of the final designed microstrip band stop filter based on metamaterial unit cell. From these results, the circuit has rejected band characterized by center frequency of $2 \mathrm{GHz}$ and it extended from 1.5 to $2.3 \mathrm{GHz}$. The insertion loss is less than $0.1 \mathrm{~dB}$ in two desired bands. Moreover, a good return loss is remarked. This confirms good transmission performances in the passbands. Besides, a power rejection capability higher than $25 \mathrm{~dB}$ is obtained and a fractional bandwidth $\mathrm{FBW}=40$ is noticed. To verify the simulated results achieved by CST microwave studio, another simulation is carried out by using accelerator driven system (ADS) electromagnetic solver. The computed results show a good agreement between both solvers.

$$
F B W=\frac{f_{1}-f_{2}}{f_{0}} \%
$$

Figure 8 displays the simulated surface current at (a) $1 \mathrm{GHz}$ and (b) $2 \mathrm{GHz}$. This analysis is done to investigating the BSF performances in the passband and stopband. The obtained surface current results at the first chosen frequency prove that there is a transmission and a displacement of the radiofrequency power between the two ports. The second chosen frequency shows that there is no current close to the output port and it is very weak in the rest of the structure, which signifies that there is no signal propagation in the designed structure. The proposed BSF circuit performances are compared with previously published filters in terms of size and rejected-band properties. It is easy to see from Table 1, that this circuit has good features that make it acceptable for many radio frequencies applications and systems.

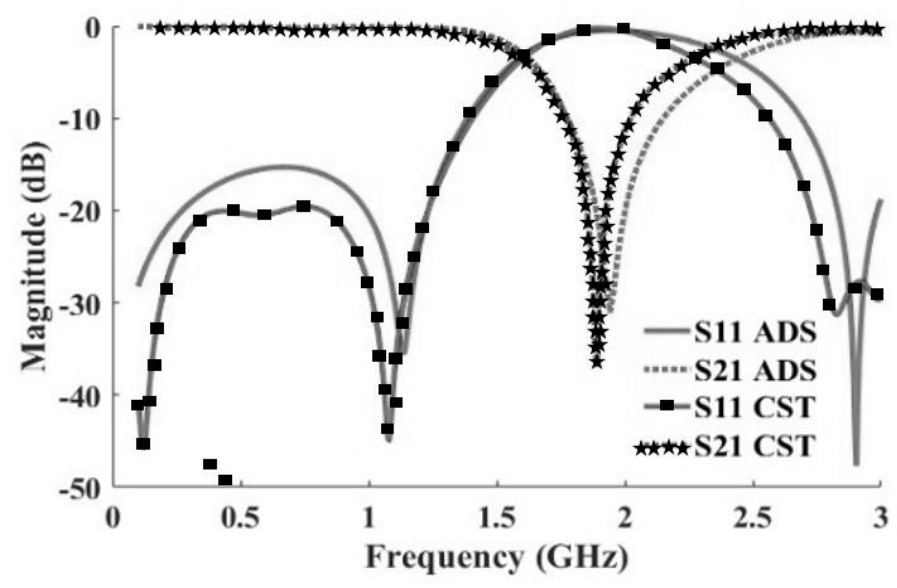

Figure 7. S-parameters results of the final designed microstrip band stop filter 

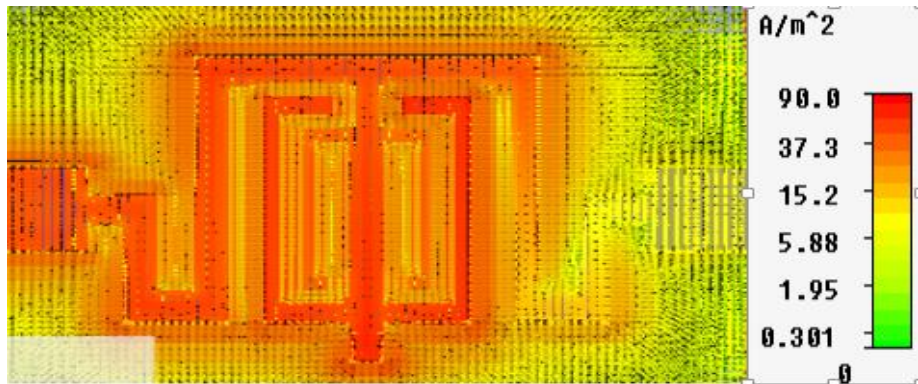

(a)
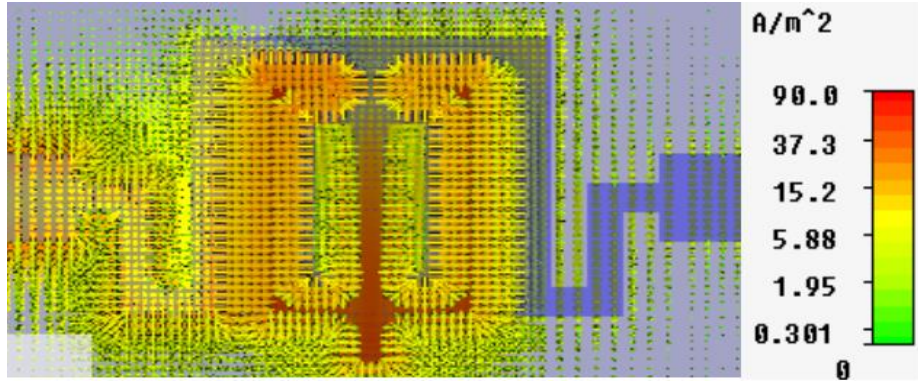

(b)

Figure 8. Simulated surface current at (a) $1 \mathrm{GHz}$ and (b) $2 \mathrm{GHz}$

Table 1. Performance's comparison

\begin{tabular}{ccccc}
\hline Parameters/Ref & Rejected Band $(\mathrm{GHz})$ & FBW & S21 deep & Size $\left(\mathrm{mm}^{2}\right)$ \\
\hline$[2]$ & {$[0.975,1.025]$} & $5 \%$ & $35 \mathrm{~dB}$ & $6370 \geq$ \\
{$[3]$} & {$[1.3,1.7]$} & $26 \%$ & $30 \mathrm{~dB}$ & 1800 \\
{$[4]$} & {$[4.4,5.4]$} & $20 \%$ & $25 \mathrm{~dB}$ & $1041 \geq$ \\
{$[1]$} & {$[2.75,3.35]$} & $20 \%$ & $25 \mathrm{~dB}$ & 609 \\
This work & {$[1.5,2.3]$} & $40 \%$ & $30 \mathrm{~dB}$ & 252 \\
\hline
\end{tabular}

\section{CONCLUSION}

In this study, a novel compact and miniature band-stop filter based on double negative metamaterial resonator was designed and optimized using CST Microwave and ADS Agilent. The filter is characterized by rejected-band between 1.5 and $2.3 \mathrm{GHz}$ which is achieved by implementing the metamaterial unit cell in the final circuit. This structure has excellent electrical features such as high return loss and insertion loss is less than $0.1 \mathrm{~dB}$. Further, it exhibits a good attenuation level in the stopband with FBW of $40 \%$.

\section{REFERENCES}

[1] K. P. Vinay, B. Ramesh, L. P, Y. E. V. Kumar, and M. M. Kumari, "Circularly polarized microstrip patch antenna using PBG structures,” Int. J. Innov. Technol. Explor. Eng., vol. 8, no. 6, pp. 491-494, 2019.

[2] E. J. Naglich, A. C. Guyette, and S. Shin, "Microwave bandstop filters with minimum through-line length," in 2015 IEEE MTT-S International Microwave Symposium, May 2015, pp. 1-4, doi: 10.1109/MWSYM.2015.7166739.

[3] R. Ranjan, A. K. Tiwary, and N. Gupta, "Design and development of bandstop filter using spiral stubs," in 2014 International Conference on Advances in Computing, Communications and Informatics (ICACCI), Sep. 2014, pp. 1360-1363, doi: 10.1109/ICACCI.2014.6968307.

[4] J. Carver, V. Reignault, and F. Gadot, "Engineering of the metamaterial-based cut-band filter," Appl. Phys. A, vol. 117, no. 2, pp. 513-516, Nov. 2014, doi: 10.1007/s00339-014-8694-7.

[5] A. Boutejdar and A. Omar, "Miniaturized lowpass and bandstop filters using controlled coupling of open-loop-ring defected ground structure," Microw. Opt. Technol. Lett., vol. 52, no. 11, pp. 2575-2578, Nov. 2010, doi: 10.1002/mop.25527.

[6] R. El Arif, M. A. Muslim, and S. H. Pramono, "Compact stepped impedance resonator bandpass filter with tunable transmission zeros," TELKOMNIKA (Telecommunication Comput. Electron. Control., vol. 15, no. 4, pp. 1689-1692, Dec. 2017, doi: 10.12928/telkomnika.v15i4.6695.

[7] M. Esmaeili and J. Bornemann, "Microstrip bandstop filters using L- and T-shaped resonators," in 2015 Asia-Pacific Microwave Conference (APMC), Dec. 2015, pp. 1-3, doi: 10.1109/APMC.2015.7411622.

[8] A. Boutejdar, "A new approach to design compact tunable BPF starting from simple LPF topology using a single T-DGSresonator and ceramic capacitors," Microw. Opt. Technol. Lett., vol. 58, no. 5, pp. 1142-1148, May 2016, doi: 10.1002/mop. 29757.

[9] G. Immadi, M. V Narayana, A. Navya, Y. D. S. Sairam, and K. Shrimanth, "Design and analysis of micro strip circular ring band 
stop filter," Int. J. Eng. Adv. Technol., vol. 8, no. 4, pp. 788-790, 2019.

[10] S.-G. Mao and Y.-Z. Chueh, "Coplanar waveguide bandpass filters with compact size and wide spurious-free stopband using electromagnetic bandgap resonators," IEEE Microw. Wirel. Components Lett., vol. 17, no. 3, pp. 181-183, Mar. 2007, doi: 10.1109/LMWC.2006.890461

[11] M. Bhaskar, J. Jasmi, and T. Mathew, "Microstrip bandstop filters based on hexagonal complementary split ring resonators," in 2015 Fifth International Conference on Advances in Computing and Communications (ICACC), Sep. 2015, pp. 311-313, doi: 10.1109/ICACC.2015.72.

[12] M. Pourbagher, J. Nourinia, and C. Ghobadi, "Compact broad band-stop filter with circular fractal-shaped stubs for X-band radar applications,” Appl. Comput. Electromagn. Soc. J., vol. 32, no. 1, pp. 56-59, 2017.

[13] K. Annam, S. Kumar Khah, S. Dooley, C. Cerny, and G. Subramanyam, "Experimental design of bandstop filters based on unconventional defected ground structures," Microw. Opt. Technol. Lett., vol. 58, no. 12, pp. 2969-2973, Dec. 2016, doi: 10.1002/mop.30192.

[14] B. Nasiri, A. Errkik, J. Zbitou, A. Tajmouati, L. Abdellaoui, and M. Latrach, "A miniature microstrip BSF using complementary split ring resonator,” Int. J. Intell. Eng. Syst., vol. 11, no. 3, pp. 29-36, Jun. 2018, doi: 10.22266/ijies2018.0630.04.

[15] B. Nasiri, A. Errkik, J. Zbitou, A. Tajmouati, L. El Abdellaoui, and M. Latrach, "A compact planar low-pass filter based on SRRmetamaterial," Int. J. Electr. Comput. Eng., vol. 8, no. 6, pp. 4972-4980, Dec. 2018, doi: 10.11591/ijece.v8i6.pp4972-4980.

[16] Y. Gaurav and R. K. Chauhan, "A compact UWB BPF with a notch band using rectangular resonator sandwiched between interdigital structure," Int. J. Electr. Comput. Eng., vol. 7, no. 5, pp. 2420-2425, Oct. 2017, doi: 10.11591/ijece.v7i5.pp24202425 .

[17] K. Jang, S. Kahng, J. Jeon, and Q. Wu, "Compact dual-band three-way metamaterial power divider with a hybrid CRLH phaseshift line," J. Electromagn. Eng. Sci., vol. 14, no. 1, pp. 15-24, Mar. 2014, doi: 10.5515/JKIEES.2014.14.1.15.

[18] A. Turkeli, A. K. Gorur, E. G. Sahin, and A. Gorur, "Design of dual wideband bandpass filter using stub loaded multimode resonators," Microw. Opt. Technol. Lett., vol. 60, no. 10, pp. 2370-2374, 2018, doi: 10.1002/mop.31393.

[19] B. Nasiri, A. Ennajih, A. Errkik, J. Zbitou, and M. Derri, "Band-pass filter based on complementary split ring resonator," TELKOMNIKA (Telecommunication Comput. Electron. Control., vol. 18, no. 3, p. 1145, Jun. 2020, doi: 10.12928/telkomnika.v18i3.13929.

[20] A. Ennajih, B. Nasiri, J. Zbitou, A. Errkik, and M. Latrach, "A novel design of miniaturized tag antenna loaded metamaterial spiral split ring resonator," Int. J. Intell. Eng. Syst., vol. 12, no. 2, pp. 12-21, Apr. 2019, doi: 10.22266/ijies2019.0430.02.

[21] B. Nasiri, A. Ennajih, A. Errkik, J. Zbitou, and M. Derri, "Analysis and design of a new miniature microstrip BPF based on metamaterial and dumbbell DGS," Int. J. Microw. Opt. Technol., vol. 15, no. 1, pp. 26-33, 2020.

[22] A. Kumar, J. Mohan, and H. Gupta, "Novel metamaterials with their applications to microstrip antenna," Int. J. Microw. Opt. Technol., vol. 11, no. 3, pp. 188-195, 2016.

[23] V. G. Veselago, "The electrodynamics of substances with simultaneously negative values of \$e\$ and \$ $\$$," Sov. Phys. Uspekhi, vol. 10, no. 4, pp. 509-514, Apr. 1968, doi: 10.1070/PU1968v010n04ABEH003699.

[24] J. B. Pendry, A. J. Holden, W. J. Stewart, and I. Youngs, "Extremely low frequency plasmons in metallic mesostructures," Phys. Rev. Lett., vol. 76, no. 25, pp. 4773-4776, Jun. 1996, doi: 10.1103/PhysRevLett.76.4773.

[25] J. B. Pendry, A. J. Holden, D. J. Robbins, and W. J. Stewart, "Magnetism from conductors and enhanced nonlinear phenomena," IEEE Trans. Microw. Theory Tech., vol. 47, no. 11, pp. 2075-2084, 1999, doi: 10.1109/22.798002.

[26] R. A. Shelby, D. R. Smith, and S. Schultz, "Experimental verification of a negative index of refraction," Science (80-. )., vol. 292, no. 5514, pp. 77-79, Apr. 2001, doi: 10.1126/science.1058847. 